\title{
Conocimientos y prácticas sobre la prevención y el control de la influenza AH1N1 en una comunidad de Floridablanca, Santander
}

\author{
María Paula Sarmiento, Oliverio Suárez, Jesús Antonio Sanabria, Cristhian Eduardo Pérez, \\ Laura del Pilar Cadena, María Eugenia Niño \\ Programa de Medicina, Facultad de Ciencias de la Salud, Universidad Autónoma de Bucaramanga, \\ Bucaramanga, Colombia
}

Introducción. La influenza AH1N1 generó una pandemia durante el año 2009, para la cual los gobiernos de todo el mundo desarrollaron medidas de mitigación y control de la propagación. En el departamento de Santander se pusieron en práctica planes de prevención orientados a la comunidad.

Objetivo. Evaluar los conocimientos y prácticas de la población sobre la prevención y control de la pandemia de influenza AH1N1.

Materiales y métodos. Se trató de un estudio transversal con muestreo no probabilístico de encuesta diseñada por los investigadores y diligenciada por personal capacitado sobre el tema. Se realizaron análisis univariado, bivariado y regresión logística.

Resultados. La muestra obtenida fue de 340 habitantes, los cuales se encontraban en su domicilio el día de la encuesta; de ellos, 97,4 \% de la población conocía sobre la pandemia. El lavado de manos y el uso de tapabocas fueron las medidas más escuchadas. Se encontró relación entre el sexo y el nivel de conocimientos, las medidas escuchadas y los medios de difusión. El nivel de escolaridad está relacionado con las medidas de control de síntomas. La edad está relacionada con la duración del lavado de manos y el uso de la cuarentena.

Conclusión. La población estudiada presenta niveles aceptables de conocimientos y prácticas de prevención de la influenza $\mathrm{AH} 1 \mathrm{~N} 1$. Se recomienda continuar con los planes de mitigación a nivel gubernamental para evitar la expansión de la influenza.

Palabras clave: subtipo $\mathrm{H} 1 \mathrm{~N} 1$ del virus de la influenza $\mathrm{A}$; brote de enfermedades; prevención y mitigación; conocimientos, actitudes y práctica en salud; distribución espacial de la población; Floridablanca, Santander.

Knowledge and practices about the prevention and the control of the influenza A H1N1 in the community of Floridablanca, Santander

Introduction. The influenza A H1N1 generated a pandemic during 2009; governments around the world developed mitigation and control strategies to contain its spread. In Santander prevention plans in a local community were put into practice.

Objectives. The knowledge and practices of the population were assessed with respect to the prevention and control of pandemic influenza A H1N1.

Materials and methods. A cross-sectional study of a random sample of processed survey designed by one of the investigators, was conducted by personnel experienced with the survey method. Analysis was by univariate, bivariate and logistic regression methods.

Results. The sample obtained was of 340 inhabitants. Nearly the entire population (97.4\%) were aware of the pandemic. Hand washing and use of surgical masks were the best known preventative actions. An association was seen between gender and level of knowledge, and between knowledge of preventative action and the media exposure. The educational level was related to symptom control measures. Age was related with the duration of hand washing and the use of quarantine.

Conclusions. The community appeared to have acceptable levels of knowledge and practices for the prevention of influenza A H1N1. Continued mitigation plans at government level were recommended to prevent the spread of influenza.

Key words: Influenza A virus, H1N1 subtype; disease outbreaks, prevention and mitigation; health knowledge, attitudes, practice; residence characteristics. 
El virus de la influenza AH1N1 pertenece a la familia Ortomixoviridae, género virus de la influenza; éste posee una nucleocápside helicoidal y una envoltura con proyecciones, las cuales están conformadas por dos glicoproteínas: la hemaglutinina y la neuroaminidasa. Según el tipo de estas glicoproteínas, serán las características antigénicas $y$, por lo tanto, su capacidad de virulencia, lo que determina su agresividad y la capacidad del huésped de reaccionar ante ellas $(1,2)$.

El genoma del virus de la influenza es ARN de cadena sencilla, polaridad negativa y segmentado, lo cual facilita el desarrollo de nuevas cepas por mutación y reorganización de los segmentos genéticos entre las distintas cepas humanas y animales del virus; esta inestabilidad genética es la responsable de la actual pandemia. Esta nueva variante fue clasificada por la Organización Mundial de la Salud (OMS) como una pandemia de tipo 6, lo que significa que involucra la aparición de brotes comunitarios ocasionados localmente sin la presencia de una persona infectada proveniente de la región del brote inicial (3).

Los primeros signos de infección por el virus AH1N1 consisten en un cuadro gripal, con fiebre, tos, cefalea, dolores musculares y articulares, dolor de garganta, rinorrea y, en ocasiones, vómito. La información obtenida de numerosos lugares afectados por brotes demuestra que el virus pandémico $A H 1 N 1$ se ha arraigado rápidamente y es hoy la cepa de virus gripal dominante en la mayor parte del mundo (4-6).

Según la OMS, hasta el 15 de noviembre de 2009, en todo el mundo se habían documentado más de 526.060 casos confirmados y alrededor de 6.770 muertes. En los 35 países de las Américas se han confirmado 190.765 casos, incluidas 4.806 defunciones. Hasta el 24 de noviembre, en Colombia se habían reportado 3.174 casos confirmados del virus $\mathrm{AH} 1 \mathrm{~N} 1$, con 160 fallecimientos; además, se ha confirmado la circulación del virus en 31 departamentos, 4 distritos y 188 municipios. En el departamento de Santander se han informado 7.936 casos, de los cuales, 99 se confirmaron, incluidos 9 casos de muerte $(7,8)$.

\section{Correspondencia:}

Laura del Pilar Cadena, Calle 157 № 15-55, Cañaveral Parque, Bucaramanga, Colombia.

Teléfono: (577) 643 6261; fax: (577) 6399147

Icadena@unab.edu.co

Recibido: 12/03/10; aceptado:02/10/10
Dado que este virus tiene una elevada tasa de transmisión, la comunidad juega un papel importante en la prevención y en el control de esta pandemia; esto se debe a que las formas más efectivas de evitar la propagación y la transmisión de la enfermedad son métodos sencillos que requieren la participación activa de la comunidad, de los cuales, se puede resaltar el adecuado conocimiento sobre qué es la infección por influenza AH1N1, cómo se contagia, cómo prevenir este contagio, los factores que lo predisponen y las diversas recomendaciones que se deben seguir para mitigar la pandemia (4-6).

Esta información se divulga por diversos medios (radio, televisión, internet, periódicos, revistas, folletos, etc.), en el marco de las acciones intersectoriales que realiza cada gobierno (7); en el caso de Santander, es el plan Santander contra la influenza, Comité Departamental Antipandemia. Éste se basa en el desarrollo de medidas de prevención y mitigación de la influenza mediante circulares, capacitaciones y diversas actividades que repercuten en áreas vitales de la atención y el control de la influenza; sus programas están dirigidos a diversos municipios e instituciones de salud del departamento de Santander, con información nacional y regional (9). Además de la educación masiva, es necesario que la comunidad se apropie del conocimiento y desarrolle actitudes y prácticas de prevención; este concepto se define como compliance, el cual se debe evaluar para determinar el impacto en la población de las estrategias de control de la pandemia.

En Colombia no se habían realizado estudios en los cuales se tuviera en cuenta la opinión, los conocimientos y las prácticas de la población como factor decisivo para mitigar la pandemia. Un estudio de esta índole es de gran ayuda para fortalecer y solucionar diversos factores que tienen gran importancia en el control de la enfermedad; por lo tanto, el objetivo del presente estudio fue evaluar el conocimiento de los ciudadanos con respecto a la enfermedad y las prácticas realizadas por la población ante esta pandemia que se encuentra latente.

\section{Materiales y métodos}

Se llevó a cabo un estudio descriptivo y transversal, utilizando una encuesta diligenciada por estudiantes del curso de Determinantes en salud (línea de Salud Pública) del programa de Medicina de la Universidad Autónoma de Bucaramanga, quienes fueron entrenados para esta actividad. 
La población de estudio estuvo constituida por hombres y mujeres, mayores de 18 años, que aceptaron participar de forma voluntaria y que eran residentes de cinco barrios de estrato medio del municipio de Floridablanca.

La selección de los barrios se hizo por conveniencia, ya que, históricamente, la universidad ha trabajado con esta población y sus habitantes están sensibilizados. Sin embargo, en la representación geográfica del municipio de Floridablanca, estos barrios reflejan la dinámica de la población de estrato medio (10).

Al llegar a las casas, los estudiantes informaban el objetivo de la actividad, invitaban a participar a un habitante mayor de edad por cada casa, le diligenciaban la encuesta y, al finalizar, se les reforzaban las medidas de prevención, haciendo énfasis en el lavado de manos, el control y la consulta rápida ante los signos de alarma y el aislamiento; después de esto les entregaban el volante emitido por el Comité Departamental Antipandemia (9). Esta selección la hicieron hasta completar encuestas en todas las casas de los barrios.

El cuestionario constaba de 22 preguntas de selección múltiple con respuesta única o múltiple, que fue elaborado por los autores del estudio; en él se evaluaban los conocimientos, entendidos como los constructos teóricos básicos sobre la enfermedad y sus síntomas; las actitudes, evaluadas como la credibilidad en la pandemia, y las prácticas, es decir, las acciones que realizaba la población para controlar los síntomas y evitar la propagación.

Las variables incluidas fueron las siguientes.

- Datos personales: barrio, estrato, edad, sexo y escolaridad

- Medidas de prevención y medios de comunicación: medidas escuchadas y medio por el cual se informó

- Lavado de manos: duración del lavado y el momento del día o circunstancia en el cual lo realizaba

- Uso de tapabocas: si estaba haciendo uso de éste; si esta respuesta era afirmativa, en qué momentos lo hacía, modo de desecharlo y la duración de su uso

- Manejo de la gripa: en caso de presentar tos, qué precauciones tomaba para no infectar a otros, control de los síntomas de la gripa, signos de alarma de AH1N1 conocidos y cuándo debía asistir al médico
- La percepción de la pandemia: si se consideraba la pandemia como real o manipulación, forma de actuar de Colombia y Santander

- Nexo epidemiológico con casos importados: si algún miembro de la familia en el último mes había viajado a Estados Unidos o México, la presencia de algún síntoma respiratorio en algún miembro de la familia.

El trabajo de campo se hizo el 27 de mayo de 2009, momento en el cual ya estaba establecida la categoría de pandemia, y se llevaban a cabo los planes de información y divulgación a la comunidad por diferentes medios.

\section{Consideraciones éticas}

Esta investigación es una encuesta de población considerada como un estudio sin riesgos (11), la cual fue avalada por el decanato y el Comité de Ética de la Facultad de Ciencias de la Salud de la Universidad Autónoma de Bucaramanga, y realizada por los estudiantes del curso de Determinantes en salud (línea de Salud Pública), en el transcurso del primer semestre de 2009.

\section{Análisis estadístico}

La captura de los datos y el análisis estadístico de la información se llevaron a cabo mediante el programa Epi-Info, versión 3.5.1, de 2008 (12).

En el análisis descriptivo para las variables cuantitativas, se aplicaron la media y la desviación estándar (DE) y, para las variables cuantitativas no paramétricas, se utilizaron la mediana y el rango.

Enelanálisis bivariado, secalcularon losestadísticos ji al cuadrado, ANOVA y odds ratio (OR) con un intervalo de confianza del $95 \%$, para establecer la relación entre sexo, edad y escolaridad con el nivel de conocimientos de la población acerca de la pandemia.

No se calcularon razones de prevalencia porque el tipo de muestreo sólo permite hallar razones de exposición.

Se realizó una regresión logística no condicional, tomando las variables de conocimientos sobre la pandemia que tuvieran un valor de significancia menor de 0,05, con ajustes por escolaridad, edad y sexo.

\section{Resultados}

Los barrios encuestados eran en su mayoría de estrato $3(95 \%)$ y 4 (5\%); las viviendas se encontraban en un estado aceptable, con 
condiciones básicas de salubridad: con servicios de agua, electricidad y recolección de basuras, entre otros. Había dos centros de salud con información sobre la influenza, una iglesia, varios parques recreativos y mercados pequeños. Históricamente, ha sido una zona residencial con adecuadas condiciones para los habitantes.

Con respecto al acceso a la información de la pandemia, en todas las casas visitadas se contaba con medios de comunicación audiovisuales (radio o televisión). No se evaluó el acceso a internet domiciliario, pero había varios establecimientos que prestaban este servicio cerca de la población estudiada. Tampoco se evaluó si en los colegios de los hijos se habían hecho campañas educativas para propagar información sobre el control de la pandemia.

Se entrevistaron 340 personas que tenían entre 18 y 82 años, con una mediana de 37 años y un rango intercuartil (RIQ) de 28,197 personas (57,9\%) eran mujeres, $219(64,4 \%)$ tenían nivel de escolaridad primaria o secundaria, 111 (32,4\%), nivel técnico o superior y sólo 10 (2,9\%) eran analfabetas.

De la población, 331 (97,4 \%) personas habían escuchado medidas de prevención contra la gripa AH1N1. Las más reconocidas fueron: el lavado de manos, conocida por 313 personas $(94,5 \%)$; el uso de tapabocas, por 312 (91,7\%); permanecer en casa, por 221 (65\%), y no saludar de mano, por 220 (64,7\%). Se agruparon en básicas: lavado de manos, uso de tapabocas, permanecer en casa y no saludar de mano, conocidas por 94 personas $(28,4$ $\%)$, y complementarias, alguna de las siguientes medidas: consumo de antivirales, uso de guantes, vacunación y consumo de vitamina $\mathrm{C}$, conocidas por 237 personas $(71,6 \%)$.

Los medios más eficaces para la promoción de las medidas fueron los noticieros, conocidos por 335 personas $(95,6 \%)$ y las propagandas televisivas, conocidas por 202 personas (59,7\%). Los menos frecuentes fueron la consulta médica, conocida por 65 personas $(19,1 \%)$ y las EPS, conocidas por 58 (17,1\%).

Se hicieron preguntas sobre el lavado de manos: 205 personas $(60,2 \%)$ dijeron gastar menos de un minuto en el lavado de manos, 100 (29,4\%) empleaban un minuto y 35 (10,3\%) señalaron que más de un minuto. En esta comunidad se encontró que 318 individuos $(93,5 \%)$ se lavaban las manos antes de comer, $278(82,1 \%)$ lo hacían después de comer, 148 (43,5\%) después de estornudar,
$113(33,8 \%)$ después de toser, 105 (30,6 \%) después de saludar a alguien con gripa, y 75 (22,1 $\%)$ después de manipular un tapabocas.

Con respecto al uso y el manejo del tapabocas, 33 personas $(9,7 \%)$ afirmaron estar utilizando tapabocas en el momento de la encuesta. De éstos, $11(33,33 \%)$ lo hacían constantemente, $15(45,5 \%)$ lo hacían dentro de la casa y $7(22,2$ $\%$ ) lo utilizaban para salir a la calle. De los 340 encuestados, 319 (93,8 \%) afirmaron que debía reemplazarse cuando se ve sucio o húmedo, 245 (72 \%) afirmaron que debía utilizarse por menos de un día y $216(63,4 \%)$ dijeron que se debía botar en la basura.

Al indagar sobre los síntomas de la gripa, 118 $(34,7 \%)$ dijeron toser en un pañuelo de tela, 114 $(33,5 \%)$, en pañuelo desechable, $97(28,5 \%)$, en tapabocas, $65(19,1 \%)$, al aire y $50(14,7 \%)$, en ropa o cuerpo. Para el control de los síntomas leves, la mayoría de la población, 202 (59,4 \%), refirió hacer uso de medidas caseras, 77 personas $(22,6 \%)$, de la farmacia, y 71 personas $(20,9$ $\%)$, de la automedicación. Sobre los signos de alarma de la presente infección por AH1N1, 298 personas $(87,6 \%)$ dijeron reconocer la fiebre alta; $36(69,4 \%)$, la dificultad para respirar; $210(61,8$ $\%)$, la tos; 173 (50,9\%), el vómito; 153 (45\%), la deshidratación; 121 (35,6\%), la fiebre leve, y 96 personas $(28,2 \%)$, la somnolencia. Las situaciones más relevantes en las que las personas afirmaron que se debía asistir al médico fueron: si la fiebre era muy alta o incontrolable, 254 personas $(74,7$ $\%)$, y si había dificultad para respirar diferente a congestión nasal, 195 personas (57,4\%). La menos importante fue: siempre, tan pronto como se iniciaran los síntomas, 95 personas $(27,9 \%)$.

Sobre la percepción de la pandemia se encontró que 226 personas $(66,4 \%)$ consideraban que la pandemia actual era real, mientras que 114 personas $(33,6 \%)$ afirmaron que era producto de la manipulación. A 250 personas $(73,5 \%)$ les parecía que el gobierno colombiano había actuado de forma adecuada ante la pandemia y 179 personas $(52,5 \%)$ creían que el departamento de Santander había tomado las medidas necesarias para el control.

Con respecto al nexo epidemiológico, se encontró que sólo 15 personas $(4,4 \%$ de la población) presentaban un familiar que hubiera viajado a Estados Unidos o México en el último mes y 90 $(26,6 \%)$ reportaron que algún miembro de su familia había tenido sintomatología respiratoria. 
En el análisis bivariado se encontró asociación entre sexo (cuadro 1), escolaridad (cuadro 2) y edad sin agrupar (cuadro 3), con los conocimientos, actitudes y prácticas de la población. De igual forma, se hizo el análisis multivariado por regresión logística no condicional, en el que la variable de desenlace era el nivel de conocimientos básicos o básicos y complementarios sobre prevención de la pandemia y, las variables de exposición, el sexo, la edad y el nivel de escolaridad. Sólo el sexo mostró una relación estadísticamente significativa, como se observa en el cuadro 4.

\section{Discusión}

Sobre los resultados obtenidos, se puede decir que el nivel de conocimiento sobre la pandemia y las medidas básicas de su prevención, fue considerablemente alto en la población estudiada y alcanzó niveles superiores al $90 \%$. La televisión fue la principal forma de educación a la población. Es llamativo que la mayoría de las personas decía estornudar o toser en un medio físico diferente a las manos o el cuerpo, así como la utilidad del lavado de manos como medida de protección; sin embargo, la mayoría de los encuestados no hacía un uso correcto de éste, tanto en tiempo de lavado, como en la pertinencia de las situaciones que lo requieren, lo cual hace pensar que el mensaje no impactó de forma completa.

En la población se observa un buen conocimiento sobre los síntomas de la enfermedad y del

Cuadro 1. Asociaciones entre sexo y conocimientos acerca de la pandemia y las medidas de protección.

\begin{tabular}{|c|c|c|c|c|}
\hline \multirow[t]{2}{*}{ Variables } & \multicolumn{2}{|c|}{ Sexo } & \multirow[t]{2}{*}{ OR } & \multirow[t]{2}{*}{ IC $95 \%$} \\
\hline & $\begin{array}{c}\text { Femenino } \\
(n=197)\end{array}$ & $\begin{array}{c}\text { Masculino } \\
(n=143)\end{array}$ & & \\
\hline \multicolumn{5}{|l|}{ Medida escuchada } \\
\hline Lavado de manos & 190 & 123 & 4,41 & $1,81-10,74$ \\
\hline Permanecer en casa & 146 & 75 & 2,59 & $1,64-4,10$ \\
\hline No saludar de mano & 138 & 82 & 1,74 & $1,10-2,72$ \\
\hline \multicolumn{5}{|l|}{ Medio por el cual escuchó las medidas } \\
\hline Amigos & 61 & 42 & 1,65 & $1,02-2,67$ \\
\hline EPS & 37 & 21 & 2,18 & $1,17-4,06$ \\
\hline \multicolumn{5}{|l|}{ Momento en el cual lava las manos } \\
\hline Después de comer & 184 & 136 & 1,93 & $1,10-3,38$ \\
\hline Después de estornudar & 91 & 56 & 1,84 & $1,18-2,86$ \\
\hline Después de toser & 70 & 45 & 2,42 & $1,49-3,91$ \\
\hline Después de saludar a alguien con gripa & 55 & 49 & 1,69 & $1,04-2,73$ \\
\hline \multicolumn{5}{|l|}{ Uso del tapabocas } \\
\hline Utilizar el tapabocas por más de un día & 57 & 39 & 1,88 & $1,12-3,16$ \\
\hline \multicolumn{5}{|l|}{ Lugar donde tose } \\
\hline Toser en un pañuelo de tela & 78 & 40 & 0,5 & $0,31-0,78$ \\
\hline Toser en un pañuelo desechable & 66 & 48 & 2,1 & $1,30-3,38$ \\
\hline \multicolumn{5}{|l|}{ Medidas para el control de los síntomas } \\
\hline Medidas caseras & 115 & 87 & 1,61 & $1,03-2,49$ \\
\hline \multicolumn{5}{|l|}{ Signos de alarma } \\
\hline Fiebre & 68 & 53 & 1,63 & $1,03-2,59$ \\
\hline Vómito & 97 & 76 & 1,81 & $1,17-2,80$ \\
\hline Tos & 125 & 85 & 2,03 & $1,30-3,17$ \\
\hline
\end{tabular}

IC $95 \%$ : intervalo de confianza del $95 \%$

Cuadro 2. Asociaciones entre nivel de escolaridad y conocimientos acerca de la pandemia y sus medidas de protección.

\begin{tabular}{lcccc}
\hline Variables & \multicolumn{3}{c}{ Nivel de escolaridad } & \\
\cline { 2 - 5 } & $\begin{array}{c}\text { Básico* } \\
(\mathbf{n}=\mathbf{2 2 9})\end{array}$ & $\begin{array}{c}\text { Superior } \\
(\mathbf{n}=\mathbf{1 1 1})\end{array}$ & OR & IC 95\% \\
\hline Medidas escuchadas & 73 & 46 & 1,51 & $0,94-2,41$ \\
$\quad$ No permanecer en casa & 16 & 1 & 8,26 & $1,08-63,12$
\end{tabular}

* Básico: incluye ninguno, primaria y secundaria

** Superior: incluye técnico y universitario

IC $95 \%$ : intervalo de confianza del $95 \%$ 
Cuadro 3. Asociaciones entre la edad y conocimientos acerca de la pandemia y sus medidas de protección.

\begin{tabular}{|c|c|c|c|c|}
\hline Variables & Media & $\mathrm{DE}$ & $\mathbf{F}$ & Valor de $\mathrm{P}$ \\
\hline \multicolumn{5}{|c|}{ Duración del lavado de manos } \\
\hline 10 segundos & 36,3 & 15,45 & 1,95 & 0,12 \\
\hline 30 segundos & 40,9 & 16,55 & & \\
\hline 1 minuto & 40,6 & 16,51 & & \\
\hline 5 minutos & 35,7 & 14,54 & & \\
\hline \multicolumn{5}{|c|}{ Permanecer en casa } \\
\hline Sí & 41,12 & 16,86 & 11,11 & 0,001 \\
\hline No & 34,94 & 15,13 & & \\
\hline
\end{tabular}

DE: desviación estándar; F: prueba estadística F de Snector

Cuadro 4. Regresión logística entre conocimiento de medidas de prevención y sexo de los encuestados.

\begin{tabular}{lcccc}
\hline & Odds ratio & \multicolumn{2}{c}{ IC 95\% } & Valor de P \\
\hline $\begin{array}{l}\text { Edad } \\
\begin{array}{l}\text { Escolaridad } \\
\text { (ninguno/básico) }\end{array}\end{array}$ & 0,99 & 0,98 & 1,01 & 0,74 \\
$\begin{array}{l}\text { Escolaridad } \\
\text { (superior/básico) }\end{array}$ & 0,72 & 0,27 & 5 & 0,82 \\
$\begin{array}{l}\text { Sexo } \\
\text { Constante }\end{array}$ & 0,55 & 0,44 & 1,19 & 0,2 \\
\hline
\end{tabular}

IC $95 \%$ : intervalo de confianza del $95 \%$

momento en el que deben consultar. No obstante, existe una mala percepción sobre las medidas básicas y complementarias, ya que el $71,6 \%$ de los encuestados creía en estas últimas, a pesar de que no existe prueba de su efectividad en la prevención de la influenza. Es de resaltar que las mujeres son quienes tenían un mejor conocimiento y actitudes frente a la prevención de la pandemia.

Como una debilidad del presente estudio, se puede identificar el hecho de que esta investigación se llevó a cabo como parte del ejercicio académico del curso de Determinantes en salud, que pretendía que los estudiantes conocieran los elementos para la estructuración de una investigación epidemiológica descriptiva, lo cual puede generar errores en el muestreo y en la recolección de los datos. La redacción de este artículo fue iniciativa de un grupo de estudiantes, en la que no quisieron participar todos los que habían recolectado la información, y la elaboración de esta publicación representó una ganancia adicional para el ejercicio académico.

Durante la búsqueda bibliográfica para este artículo, no se encontró ningún artículo latinoamericano que analizara los conocimientos y las prácticas de la comunidad en torno a la infección por influenza AH1N1. En la literatura internacional existen pocos estudios que aborden este tema; se encontraron principalmente artículos sobre las mejores medidas de prevención, el diagnóstico y el manejo adecuado, y aún más pocos, sobre los conocimientos, creencias y actitudes de la población.

A nivel internacional, Eastwood et al. (13) realizaron un estudio transversal en habitantes de Australia y encontraron que sólo $23 \%$ de los 1.166 encuestados demostraron un claro entendimiento del concepto de influenza pandémica; el método más efectivo para conocer las medidas de prevención fueron el medio televisivo (31,2\%), el correo electrónico $(27,8 \%)$ y la internet $(13,9 \%)$. Las mujeres presentaban mayor cumplimiento de las recomendaciones (OR=2, IC $95 \%$ 1,3-3,1), y la ocupación fue un factor limitante para permanecer en cuarentena (independientes: $\mathrm{OR}=4,9 ; 2,3-10,5$; dependientes: OR=3,3; 2-5,4).

En la misma población australiana, específicamente del sudeste del continente, Marshall $\mathrm{H}$ et al. (14) contactaron 1.975 hogares seleccionados al azar, con el fin de analizar el conocimiento de la comunidad sobre la pandemia y observar la aceptación y aplicabilidad de las medidas propuestas. Encontraron que el 50,2 \% nunca había oído de la pandemia de influenza A H1N1, con diferencias según la edad (45 a 64 años mejor conocimiento, $p<0,001$ ), el sexo (la mujer tiene mejor conocimiento, $p=0,039$ ) y el nivel de escolaridad (a menor nivel, menor conocimiento, $\mathrm{p}=0,014)$.

Aunque las condiciones de la población australiana son diferentes a las de Floridablanca, las percepciones globales son muy parecidas, pues el sexo femenino marca una relación con el nivel de conocimientos sobre la influenza, lo cual es probable que sea producto de los roles sociales que representa el sexo.

Por otra parte, Lau et al. (15), utilizando la misma metodología de encuestas telefónicas en 550 
hogares de Hong Kong, encontraron que los habitantes presentaban muchos conceptos errados debido a la asociación entre la presente pandemia con la experiencia pasada de la influenza aviar. Ante la pregunta sobre medidas de prevención, más de $90 \%$ sabía que debe permanecer en cuarentena e informar ante la sospecha de fiebre o síntomas de gripe; sin embargo, menos de $20 \%$ de los encuestados aplicaban estas medidas.

Estos datos contrastan con el presente estudio, pues sólo el $65 \%$ considera la cuarentena como una medida de protección y el $87,6 \%$ de la población toma la fiebre alta como un signo de alarma. Sin embargo, en el presente estudio no se investigó sobre la aplicación de estas medidas. La televisión sigue siendo el mejor método de información a la comunidad; sin embargo, las diferencias culturales determinan el porcentaje de uso y la introducción de nuevos métodos masivos de comunicación, como el internet.

Rubin etal.(16), por medio de encuestas telefónicas, exploraron los niveles de ansiedad de los habitantes del Reino Unido y cómo se afectaban las medidas de prevención ante la influenza. Encontraron que la raza determina la utilización de estrategias básicas (como lavado de manos, permanecer en casa, en raza minoritaria) y las medidas de evitación (salir a espacios concurridos, viajes internacionales, en raza blanca). Además, encontraron que el haber estado informados previamente mediante folletos disminuía el nivel de ansiedad en comparación con quienes no los habían recibido $(p=0,03)$.

En Estados Unidos se realizó un estudio en el marco del Proyecto Pittsburgh de prevención de la influenza (17), cuyo objetivo era conocer las medidas no farmacológicas más utilizadas por los profesores y padres de familia de estudiantes de grados inferiores. En los resultados encontraron que las medidas más básicas y sencillas, como el lavado de manos y el cubrirse la boca al toser o estornudar, eran las más utilizadas por todos los encuestados y sus hijos; mientras que el llamado a una línea de emergencia, el uso de tapabocas o el de guantes, eran las menos frecuentemente usadas por lo dispendioso del proceso.

Aunque no es la misma población, Bravo et al. (18) midieron el nivel de conocimiento sobre la pandemia entre los trabajadores de la salud de un hospital de referencia de Lima, Perú, y encontraron que $73,5 \%$ a $77,8 \%$ de los profesionales médicos, $35,4 \%$ de los profesionales enfermeros y $64,3 \%$ de los internos, tenían un nivel de conocimiento adecuado. Llama la atención que el nivel de conocimientos y, por lo tanto, la posible capacidad de reacción ante esta pandemia, es mucho menor al esperado para el tipo de población encuestada, por lo cual surge la inquietud sobre el nivel de conocimiento que pueda tener la comunidad en general. En el presente estudio, el 97,4 \% había escuchado sobre la influenza $\mathrm{AH} 1 \mathrm{~N} 1 \mathrm{y}$, de ellos, más de $90 \%$ conocía que el lavado de manos y el uso de tapabocas eran medidas eficaces para la prevención, lo cual no significa que la comunidad las lleve a cabo.

Otro estudio interesante que, a pesar de no tratar sobre la influenzaAH1N1, analiza los conocimientos y actitudes que tiene la población inglesa ante una nueva pandemia similar a la influenza aviar, permite identificar puntos débiles y fortalezas de los programas desarrollados a nivel gubernamental para mantener a la población alerta ante nuevos brotes. Por medio de la metodología de grupos focales, Morrison y Yardley (19) encontraron que las personas tenían buen conocimiento sobre las formas de contagio de la influenza, pero no sobre la pandemia, sin encontrar diferencias por edad o por sexo. Una de las barreras para no aplicar los métodos de cuarentena fue los lazos afectivos con las posibles personas enfermas y la falta de creencia en el método como estrategia de prevención. Además, encontraron que, en el momento en que la amenaza de pandemia disminuía, la población cesaba en la aplicación de medidas de prevención pero, cuando reaparecía la amenaza, se retomaban las prácticas; los mayores de edad eran quienes las cumplían de forma más rigurosa.

En otro estudio diseñado por Raude y Setbon (20), en el que se buscaba establecer las percepciones sobre la amenaza de una pandemia de influenza en Francia, se utilizó una metodología de recolección de la información por medio de encuestas telefónicas aplicada a una población total de 374 individuos mayores de 18 años. Se encontró que más del 80 $\%$ pensaba que el virus sólo era transmitido por contacto directo de persona a persona y no era posible a través de fómites, lo cual modificaba la percepción sobre la aplicación de diversas medidas de prevención. Por último, se evidenció que en la población las medidas farmacéuticas no eran vistas como una forma adecuada para prevenir el riesgo de contraer la enfermedad o para reducirlo, contrapuesto a lo que ocurría con las medidas no 
farmacéuticas, tales como el aislamiento social y la mejora en la higiene personal (el lavado de manos y el uso de tapabocas), con una aprobación mayor de $70 \%$ de los encuestados.

Con los datos encontrados en la comunidad de Floridablanca y el análisis de la escasa literatura en el tema, es posible afirmar que el nivel de conocimientos de la población ante la pandemia de influenza AH1N1 es aceptable; sin embargo, la aplicabilidad de dicha información es precaria y requiere que se realicen más intervenciones que garanticen un mejor entendimiento de las ventajas de aplicar las medidas de prevención frente a los riesgos que conlleva el contraer la enfermedad.

Es importante resaltar que los medios masivos de comunicación, como la televisión y el internet, siguen teniendo vigencia, sobre todo para que la comunidad siempre esté alerta ante amenazas futuras de nuevas cepas de influenza de las cuales se desconozcan su comportamiento, ya que, según Eastwood et al., sólo el 15,2 \% cree que se puede volver a repetir la pandemia, y en el presente estudio el 33,6 \% consideraba la pandemia actual como una manipulación.

También, es relevante que los gobiernos desarrollen medidas de prevención y control, incluido un mejor sistema de alerta, diagnóstico y manejo integral precoz de nuevos brotes de influenza, para disminuir las posibles inequidades en la atención y el impacto general en la población. Así lo recomienda De la Hoz en su editorial (21), según los hallazgos de este estudio, en el que la población identificó como adecuadas las estrategias implementadas en $52,5 \%$ y $73,5 \%$ a nivel departamental y nacional, respectivamente.

En conclusión, con los resultados obtenidos se observa que la población analizada tiene un alto nivel de conocimientos sobre la pandemia. Se requieren más investigaciones para profundizar el grado aplicabilidad que la población presenta de las medidas de prevención ante el virus de la influenza y el tiempo que persiste esta actitud. Con ellos se podrían desarrollar campañas más costo-efectivas que integren a la población, y a los entes locales, departamentales y nacionales de salud, en la planeación y preparación ante posibles epidemias futuras.

\section{Agradecimientos}

Al decanato de la Facultad de Ciencias de la Salud de la Universidad Autónoma de Bucaramanga, por el apoyo en la realización de esta actividad.
A los estudiantes que cursaban Determinantes en salud durante el primer semestre del 2009, por su colaboración en la realización de las encuestas.

\section{Conflicto de intereses}

Expresamos que no existen conflictos de intereses de ningún tipo.

\section{Financiación}

El decanato de la Facultad de Ciencias de la Salud de la Universidad Autónoma de Bucaramanga apoyó económica, logística y materialmente la realización de este proyecto.

\section{Referencias}

1. Hay AJ, Gregory V, Douglas AR, Lin YP. The evolution of human influenza viruses. Philos Trans R Soc Lond B Biol Sci. 2001;356:1861-70.

2. Scalera NM, Mossad SB. The first pandemic of the $21 \mathrm{st}$ century: A review of the 2009 pandemic variant influenza A (H1N1) virus. Postgrad Med. 2009;121:43-7.

3. Organización Mundial de la Salud. Fase actual de alerta de pandemia según la OMS. Fecha de consulta: 1 de diciembre de 2009. Disponible en: http://www.who.int/csr/ disease/avian_influenza/phase/es/index.html.

4. Organización Mundial de la Salud. Gripe Pandémica (H1N1) 2009. Fecha de consulta: 24 de octubre de 2009. Disponible en: http://www.who.int/csr/disease/swineflu/es.

5. Ministerio de la Protección Social. $A B C$ de la influenza porcina (Gripe porcina). Documento de trabajo número 9589, abril 27 del 2009. Fecha de consulta: 24 de octubre de 2009. Disponible en: http://web.presidencia.gov.co/ sp/2009/abril/27/07272009.html.

6. Centers for Disease Control and Prevention. 2009 AH1N1 Flu. Fecha de consulta: 24 de octubre de 2009. Disponible en: http://cdc.gov/ah1n1flu.

7. Comité Departamental Antipandemia. Santander contra la influenza. Fecha de consulta: 1 de diciembre de 2009. Disponible en: http://influenza.saludsantander.gov.co.

8. Ministerio de la Protección Social. Informe de prensa No. 121 de 2009. Evolución de la situación del virus pandémico H1N1/09 en las Américas y Colombia. Fecha de consulta: 1 de diciembre de 2009. Disponible en: http:// www.minproteccionsocial.gov.co/VBeContent/NewsDetail. asp? ID=19112\&IDCompany=3.

9. Ministerio de la Protección Social. Informe de Prensa No. 120 de 2009. Minprotección y OPS lanzan caja de herramientas para manejo del AH1N1. Fecha de consulta: 1 de diciembre de 2009. Disponible en: http://www. podersobreelah1n1.com/comunicaciones.html.

10. Alcaldía de Floridablanca. Plan de Ordenamiento Territorial de Floridablanca. Fecha de consulta: mayo 10 de 2009. Disponible en:http://www.floridablancasig.com.

11. Ministerio de Salud. Resolución número 8430 de 1993. Por la cual se establecen normas, científicas, técnicas y administrativas para la investigación en salud. Bogotá: Ministerio de Salud; 1993. 
12. Centers for Disease Control and Prevention. Epilnfo version 3.5.1 de 2008. Software libre. Fecha de consulta: 20 de septiembre de 2009. Disponible en: http://www.cdc. gov/epiinfo.

13. Eastwood K, Durrheim D, Francis JL, d'Espaignet ET, Duncan S, Islam F, et al. Knowledge about pandemic influenza and compliance with containment measures among Australians. Bull World Health Organ. 2009;87:58894.

14. Marshall H, Ryan P, Roberton D, Street J, Watson $\mathbf{M}$. Pandemic influenza and community preparedness. Am J Public Health. 2009;99(Suppl.2):S365-71.

15. Lau JT, Griffiths S, Choi KC, Tsui HY. Widespread public misconception in the early phase of the $\mathrm{H} 1 \mathrm{~N} 1$ influenza epidemic. J Infect. 2009;59:122-7.

16. Rubin GJ, Amlôt R, Page L, Wessely S. Public perceptions, anxiety, and behaviour change in relation to the swine flu outbreak: Cross sectional telephone survey. BMJ. 2009;339:b2651.
17. Stebbins S, Downs JS, Vukotich CJ Jr. Using nonpharmaceutical interventions to prevent influenza transmission in elementary school children: Parent and teacher perspectives. J Public Health Manag Pract. 2009;15:112-7.

18. Bravo K, Gamarra M, Gonzales S, Lecca D, Martínez A, Mendoza M, et al. Nivel de conocimientos sobre la influenza A H1N1 en los trabajadores de salud del Hospital Nacional Arzobispo Loayza, Perú. Rev Peru Epidemiol. 2009;13:1-7.

19. Morrison LG, Yardley L. What infection control measures will people carry out to reduce transmission of pandemic influenza? A focus group study. BMC Public Health. 2009,9:258.

20. Raude J, Setbon M. Lay perceptions of the pandemic influenza threat. Eur J Epidemiol. 2009;24:339-42.

21. De la Hoz F. Influenza pandémica 2009-2010, ¿En qué podemos mejorar la respuesta? Biomédica. 2009;29:337-8. 